\title{
Study of Optical Cloaking Lenses Design for Optimizing Large Vehicle Driver's Visibility in Bandar Lampung
}

\author{
O. F. Tri Maryana ${ }^{1}$, L. Y. Ginting ${ }^{2}$, F. Pahlawan ${ }^{3}$, M. Y. Darmawan ${ }^{4}$ \\ 1,3,4 Physics Department, Institut Teknologi Sumatera J1. Way Hui, Lampung Selatan, 40132, \\ Indonesia. \\ ${ }^{2}$ Engineering Physics Department, Institut Teknologi Sumatera Jl. Way Hui, Lampung Selatan, \\ 40132, Indonesia.
}

E-mail: okky.trimaryana@fi.itera.ac.id

\begin{abstract}
Nowadays, invisibility efforts by scientists and engineers have focused on building a large broadband, multidirectional cloaking device. The device is usually a cloak that works in macroscopic dimensions and for the entire visible spectrum. Addition with the full-field of light, no new materials, simple infrastructure, and scale easily may be considered an 'ideal' cloaking device. This paper report of implementing ray optics cloaking formalism presented previously by Choi and Howell [1] using lenses design for cloaking arbitrarily large, and moving objects such as large vehicle. We apply their formalism using simulation and experiment scheme to general optical systems up to four convex lenses. The results support the information to create optical cloaking prototype for optimizing large vehicle driver's visibility as alternative solution for decreasing traffic accident in Bandar Lampung.
\end{abstract}

\section{Introduction}

Cloaking area is an area where any object placed on the area will become invisible. Initial ideas of cloaking area was conducted for theoretical framework to create a curved space for electromagnetic fields, an effort by carefully constructing materials using coordinate transformations. Cloaking device is a system that combines transformation theory and illusion technology. In principle, cloaking devices aim to totally or partly hide objects, so that the objects will disappears from the view. There several types of cloaking devices are electromagnetic cloaking, spatial cloaking, space-time cloaking, and many more as described by Choi \& Howel $[1,2]$.

Choi \& Howell reported a ray optics cloak that is designed for continuously multidirectional angles in three dimensions model. The demonstration is the first isotropic materials device system, for transmitting rays in the visible spectrum regime. They also provide an effective formalism, using ray optics approximation, to describe model of perfect optical cloaks in the small-angle or paraxial limit. Those formalisms can apply to general optical systems up to four lenses design, and show what systems can be considered as 'perfect' paraxial cloaks for ray optics. This is the first technique in laboratorium field utilizing visible light and simple integrated setup. 


\section{Theory}

In the study of optical cloaking, there are several variables that must be considered, including the type of lens, the size of the lens that used. These variables are influential in building optical cloaking systems, especially in $4 \mathrm{f}$ systems (4 lenses). Figure 1 describes cloaking area four lens configuration.

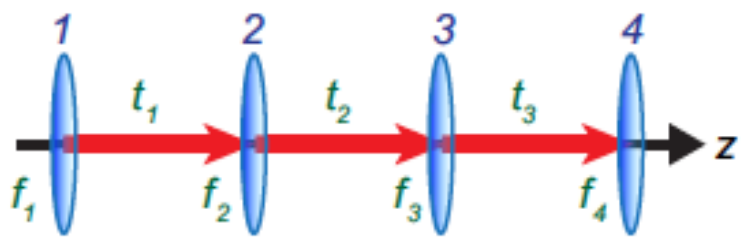

Figure 1. Area of paraxial optical cloaking for four lenses system [2].

Choi \& Howell succeeded in developing formalism using geometric optical concepts, in this case calculating paraxial approximations. In the first-order approach called the "partial approach," light rays are considered to deviate slightly from the center of the system axis. This is known as the small angle approach. The propagation of light rays through the optical system can be explained by the matrix method 'ABCD'. Because of 'perfect' cloaking systems only replicate the medium through ambient volume, the ABCD matrix is only considered a 'translation matrix'.

The easiest non-trivial solution is only considering symmetrical rotational systems with thin lenses, and in free spaces with $n=1$. The $A B C D$ matrix for one of the thin lenses is given by equation (1)

$$
\left[\begin{array}{ll}
\mathrm{A} & \mathrm{B} \\
\mathrm{C} & \mathrm{D}
\end{array}\right]_{\text {Thin lens }}=\left[\begin{array}{cc}
1 & 0 \\
-1 / \mathrm{f} & 1
\end{array}\right] \text {, }
$$

where $f$ is the focal length of the lens that we used. We can easily see that Eq. (1) will only be satisfied if $f=\infty$ because the lens has no optical power. This has no cloaking region and no optical effect. For the following scheme, we will use f's to show the focal lengths, and t's to show the distances between the optical elements (Fig. 1) The ABCD matrix for a four lens system is done by making the second half symmetric to the first half $(\mathrm{f} 1=\mathrm{f} 4, \mathrm{f} 2=\mathrm{f} 3, \mathrm{t} 1=\mathrm{t} 3)$. We then require $\mathrm{A}=1$ and $\mathrm{C}=0$ for $\mathrm{ABCD}$ matrix. These conditions are conveniently satisfied by

$$
t_{1}=f_{1}+f_{2}
$$

With Eq. (1) the ABCD matrix becomes

$$
\left[\begin{array}{cc}
1 & f_{1}\left(-2 t_{1}^{2}+f_{1}\left(2 t_{1}+t_{2}\right)\right) /\left(f_{1}-t_{1}\right)^{2} \\
0 & 1
\end{array}\right]
$$


We can now set $B=\left(2 t_{1}+t_{2}\right)$, and solve for $t_{2}$ :

$t_{2}=2 f_{2}\left(f_{1}+f_{2}\right) /\left(f_{1}-f_{2}\right)$

We can now set, and solve for total length

$L=2 t_{1}+t_{2}=2 f_{1}\left(f_{1}+f_{2}\right) /\left(f_{1}-f_{2}\right)$

\section{Method}

The four convex lenses experimental set up which used to create the optical is shown in Figure 2.

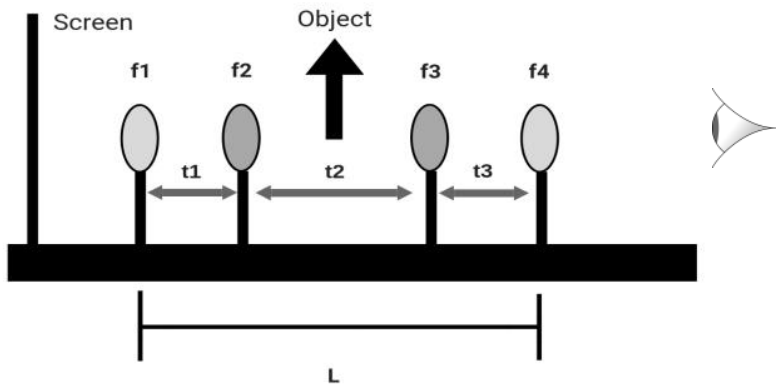

Figure 2. Experimental set up of cloaking_area system for the four convex lenses.

The set up of four convex lenses with a diameter $5 \mathrm{~cm}$ is used with focal length of 100, 50, 50 and $100 \mathrm{~mm}$ respectively. The distance between $\mathrm{f}_{1}$ and $\mathrm{f}_{2}=15 \mathrm{~cm}, \mathrm{f}_{2}$ and $\mathrm{f}_{3}=30 \mathrm{~cm}$ and $\left(\mathrm{f}_{1}-\mathrm{f}_{2}\right)=\left(\mathrm{f}_{3}-\right.$ $\mathrm{f}_{4}$ ). Figure 2 show the spacing between the lenses is the set up that produces the clearest shadow focus on the screen based on the equations. Objects (e.q: rules, fingers etc) observed are placed between the lenses arranged, then observing objects taken from the front of the lens directly. The objects placed in the center of the system or in front of the third lens seems to disappear from view. Objects that block the lens will not appear on the screen or in other words being invisible. 


\section{Results and Discussion}

The requirement of optical cloaking is to use four lenses with similar materials. The refraction of light distributed by the same size because the lenses used are the same diameter as the minimum requirement. The result of simulation of the light beam passing through the cloaking system with four lenses (4f) is shown in Figure 3.

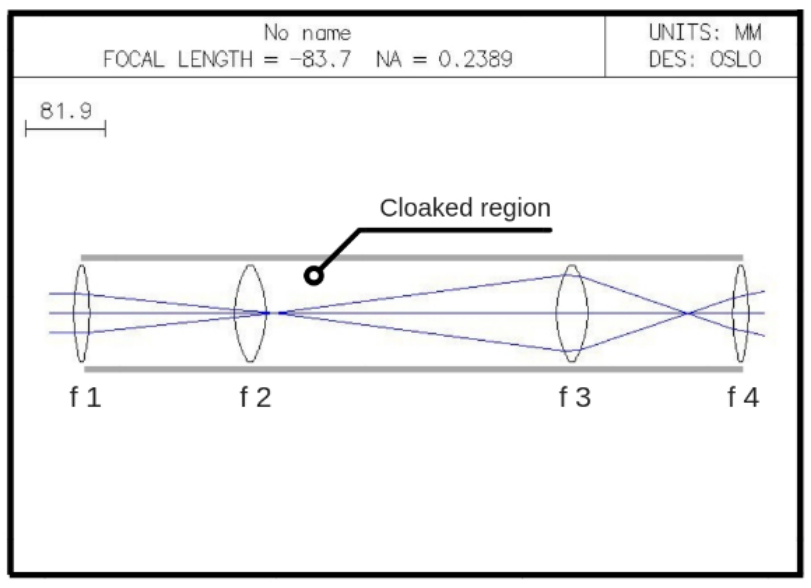

Figure 3. Simulation result of light beam on a perfect paraxial cloak that passes through four convex lenses based on setting up figure 2 . The set up of four convex lenses with a diameter $5 \mathrm{~cm}$ is used with focal length of $100,50,50$ and $100 \mathrm{~mm}$ respectively.

The set ups for this optical system are regular convex lenses based on educational laboratory framework. Unlike diverging concave lenses, convex lenses are refractory in nature. The light rays that come parallel to the axis of the lens will be biased toward the focal point. The rays can be captured on the screen and are positive. The focal length of the thicker convex lens is shorter than the length of the thin convex lens. Refracted rays in the cloaking system will conduct with the properties of a convex lens.

The area where the light beam or ray tracing does not pass through has the potential as the cloaking area as shown in figure 3. When an object is placed in the area, the object will disappear from view. In this experiment used one type of convex lens with $5 \mathrm{~cm}$ diameters comparing with same experiment by Sugiarto [3] that used thinner of convex lenses, namely $3.6 \mathrm{~cm}$ as shown in Table 1 . The distance between the lens depends on the size of the lens. The larger the diameter of the lens used then the cloaking area will be even larger_as shown in the figure 3. This result shows the dependence of vehicles dimensions with the cloaking area. The wider the cloaking area of the lenses system, the larger part of vehicle that blocks driver's visibility will be invisible. This will result in greater overall visibility for the driver.

The experimental confirmation of the result performed by chen [4] that cloaking area with $4 \mathrm{f}$ convex lenses are limited to certain areas only, not $100 \%$ of the object to be observed can disappear from the view. The limitation of cloaking area is formed around the upper third of the lens [5] diameter only in the region between ( $L 2-L 3)$, as shown in Figure 3 and confirmed with experimental result as shown in Figure 4. 


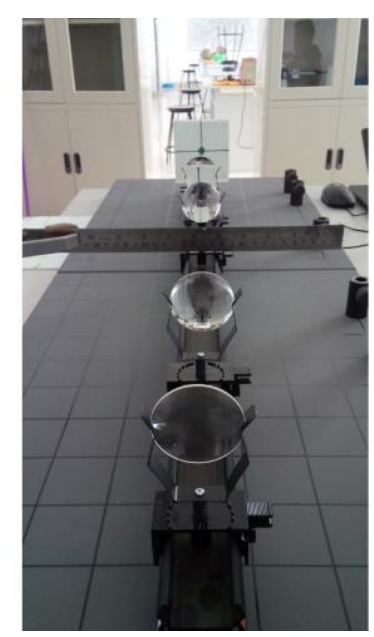

(a)

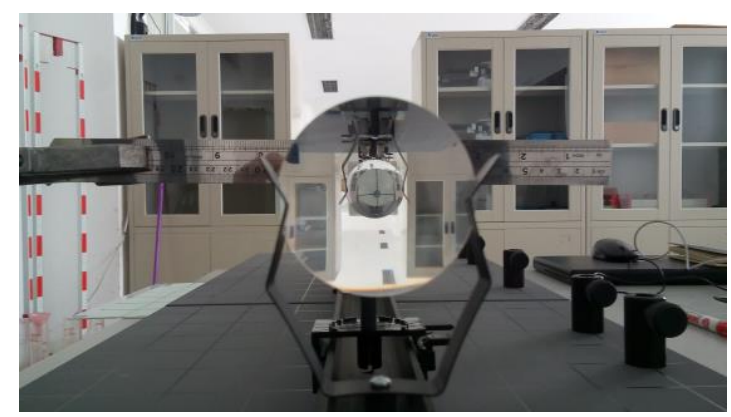

(b)

Figure 4. Experimental result of cloaking optics $4 \mathrm{f}$ convex lenses. (a) experiment set up with $30 \mathrm{x} 1$ $\mathrm{m}$ ruler, (b) Optical illution based on four lenses cloaking system.

The $4 \mathrm{f}$ convex lenses system are shown in Figure 4. In Figure 4 shows optical illusion of $4 \mathrm{f}$ system with a diameter of $5 \mathrm{~cm}$ lens. Figure $4 \mathrm{~b}$ shows that a rectangular ruler object $30 \mathrm{x} 1 \mathrm{~cm}$ in size which is placed on the upper third of the lens $( \pm 3 \mathrm{~cm})$, partially invisible when viewed from the front of the lens. This result proves that the setup of the $4 \mathrm{f}$ convex system has succeeded in forming a cloaking area. Additional information is the position of the observation angle form viewer also affects the resulting image of optical illusion. 
Table 1. Cloaking area produced by different diameter of convex lenses.

\begin{tabular}{|l|l|l|l|}
\hline $\begin{array}{l}\text { Diameter of Lens } \\
(\mathrm{cm})\end{array}$ & $\mathrm{f}_{1}=\mathrm{f}_{4}(\mathrm{~mm})$ & $\mathrm{f}_{2}=\mathrm{f}_{3}(\mathrm{~mm})$ & $\begin{array}{l}\text { Max. cloaking area } \\
(\mathrm{cm})\end{array}$ \\
\hline $3.6^{*}$ & $100^{*}$ & $50^{*}$ & \\
\hline 5 & 100 & 50 & \pm 2 \\
\hline
\end{tabular}

*: Previous work reported by Sugiarto (2018)

\section{Conclusion}

The optical cloaking design by $4 \mathrm{f}$ convex lenses can produce an optical illusion to eliminate the blocking object, in accordance with the purpose of research to enlarge driver visibility. Maximum cloaking area occurs in the intermediate area along the system. The minimum constraint of cloaking formed for the convex lens with a diameter of $5 \mathrm{~cm}$ in this experiment is $\pm 3 \mathrm{~cm}$. The distance between the lens and the cloaking area generated in this optical cloaking set depends on the amount of focal point of the convex lens used. Larger lens diameter can produce larger cloaking area that can be fitted with the blocking area on the vehicle dimension.

\section{References}

[1] J. S. Choi and J. C. Howell. Paraxial ray optics cloaking. Opt. Express, 2014, Vol. 22, $29465-$ 29478.

[2] Choi, Joseph. Practical Invisibility Cloaking. Thesis University of Rochester, New York, 2016: $12-17$.

[3] Sugiarto, I. T., et. al. Optical Illusion Design Based On Four Convex Lenses System and Cloaking Area Characterization. Jurnal Pendidikan Fisika Indonesia, 2018, 14 (1) 40-45.

[4] H. Chen, B. Zheng, L. Shen, H.Wang, X. Zhang, N. I. Zheludev, and B. Zhang. Ray-optics cloaking devices for large objects in incoherent natural light. Nat. Comm, 2013, Vol. 4, 2652.

[5] X. Z. Chen, Y. Luo, J. J. Zhang, K. Jiang, J. B. Pendry, and S. A. Zhang. Macroscopic invisibility cloaking of visible light. Nat. Comm, 2011, 2, 176.

\section{Acknowledgment}

The authors acknowledge Program Hibah Penelitian Institut Teknologi Sumatera Tahun 2018 for the support this research work. 